Sociedades Precapitalistas, vol. 7, nº 1, e021, diciembre 2017. ISSN 2250-5121

Universidad Nacional de La Plata.

Facultad de Humanidades y Ciencias de la Educación.

Centro de Estudios de Sociedades Precapitalistas (CESP)

\title{
Reevaluando la evidencia acerca de la cacería del hipopótamo en Egipto predinástico
}

\author{
Reassessing evidence concerning the hunting of hippopotami in \\ Predynastic Egypt
}

Sebastián Francisco Maydana *

* Universidad de Buenos Aires - Consejo de Investigaciones de la Universidad Nacional de Salta, Argentina I maydanasf@gmail.com

\section{PALABRAS CLAVE}

Cacería del hipopótamo

Predinástico

Arqueozoología

Simbolismo

\section{KEYWORDS}

Hippopotamus hunt

Predynastic

Archaeozoology

Symbolism

\section{RESUMEN}

El motivo de la cacería del hipopótamo aparece muy a menudo en la iconografía predinástica. Sin embargo, debido a ciertas particularidades que posee dicha representación algunos autores (Mond y Myers, 1937; Behrmann, 1996: 134-136) han sugerido que el objetivo de la expedición no era la muerte del animal sino su captura y posterior traslado a la aldea. Veinte años después, poseemos suficiente evidencia arqueológica y arqueozoológica como para volver a revisar las teorías de estos autores de forma de refutarlas o confirmarlas. Particularmente relevante para ello fue el hallazgo en Hierakonpolis de los restos de un joven hipopótamo que presenta signos de haber sido cautivo por algún tiempo en la aldea. Más allá de eso, resulta útil examinar además evidencia que no fue tenida en cuenta en el pasado, como aquella que otorgan los etnógrafos modernos y arqueozoólogos. El resultado de esta compulsa parece sugerir que la muerte del hipopótamo efectivamente sucedía en el momento de la cacería, y esto debido al peligro que conllevaría el traslado del animal vivo. Más aún, es preciso cuestionar la idea misma que las fuentes iconográficas son narraciones de hechos verdaderos. En cambio, la propuesta de este artículo será su examen en clave simbólica.

\section{ABSTRACT}

The theme of hunting the hippopotamus was often depicted in Predynastic iconography, but due to some of its particularities it has been suggested that hippopotami were, in fact, not killed but rather captured alive (Mond and Myers, 1937; Behrmann, 1996: 134-136). Twenty years have passed, and evidence both archaeological and archaeozoological has since grown significantly. We now have enough sources to reassess the corpus of evidence in order to debunk or ratify their theses. Particularly relevant to supporting her hypothesis was the finding in Hierakonpolis of a young hippopotamus' remains that show signs of having been held captive in the village. Moreover, it is helpful to examine evidence not taken into account by the author such as ethnohistorical research and the latest archaeozoological findings. The outcome of this research seems to suggest that the killing of hippopotami did in fact take place during the hunting expedition, due to the dangerous nature of transporting the living beast. Furthermore, the idea of iconographic evidence as a narration of true events should be challenged and understood instead as being of symbolic nature. 


\section{Introducción}

“y pensó, mientras alisaba el negro pelaje, que aquel contacto era ilusorio y que estaban como separados por un cristal, porque el hombre vive en el tiempo, en la sucesión, y el mágico animal, en la actualidad, en la eternidad del instante”. Jorge Luis Borges, El Sur.

Hermosas palabras para explicar que la conquista del tiempo por el hombre es también la invención de su mortalidad. Una variación de este mismo pensamiento la encontramos en un extraordinario documental de Werner Herzog sobre las pinturas rupestres de la cueva Chauvet en Francia. En él, el director alemán reflexiona sobre lo que nos separa de aquellos que inscribieron la roca hace más de treinta mil años: “nosotros estamos atrapados en la historia, ellos no lo estaban” (Herzog, 2010).

Estas ideas son tremendamente relevantes para el trabajo del egiptólogo (y probablemente para el historiador en general), ya que expresan lo que muchas veces tememos admitir: que nuestro (actual) pensamiento es histórico por naturaleza, que no podemos pensar fuera de la historia, mientras que los antiguos egipcios sí lo hacían. Buena parte de los problemas que tienen que ver con la interpretación de la iconografía y los textos egipcios antiguos derivan de este simple hecho. Los egiptólogos debemos enfrentar tales desafíos constantemente, agravados por la escasez de evidencia disponible, y por lo tanto debemos aceptar que no podemos lograr un conocimiento verdadero de cada aspecto de la civilización egipcia, sino tan sólo un entendimiento aproximado.

En este artículo quisiera, basándome en la evidencia iconográfica disponible relacionada con la cacería de hipopótamos durante el período Predinástico (Nagada I-IIc), reevaluar algunas interpretaciones propuestas por investigadores del área (Mond y Myers, 1937; Behrmann, 1996). Estos autores han especulado con la posibilidad que los animales no fueran muertos durante la cacería sino más bien capturados vivos, y conducidos hacia la aldea donde eran mantenidos en corrales o recintos (Behrmann, 1996: 135), o incluso domesticados para ser utilizados en la cacería de otros animales (Mond y Myers, 1937: 38).

Aunque al momento de formular sus hipótesis la evidencia arqueológica era relativamente escasa, este aspecto ha sido subsanado en gran parte en los últimos años, incrementando enormemente nuestro entendimiento del período nagadiense. Para empezar, la etnografía ha probado ser una herramienta valiosa a la hora de comparar sociedades diferentes e incluso separadas en el tiempo (Adler, 2007; de Heusch, 2007). En segundo lugar, la arqueología predinástica y del dinástico temprano ha mostrado una gran vitalidad en las últimas décadas. En este sentido hay que subrayar el continuado trabajo de las expediciones a sitios del Alto Egipto como Abydos (Dreyer, 1998; 2003), Hierakonpolis (Friedman, 1999; 2004), Buto (Hartung et al., 2012), etc. De allí la multiplicación de los trabajos y la información disponible acerca de este crucial período, aquel que rodea el proceso de surgimiento del Estado en el Valle del Nilo, lo cual a su vez motivó a un número creciente de historiadores (entre los cuales me cuento) a estudiar dichos procesos interpretando la nueva evidencia.

En cuanto a la cacería como práctica, sólo recientemente ha llamado la atención de los investigadores, y actualmente está siendo estudiada atentamente tanto para el predinástico (Linseele y van Neer, 2009; Hendrickx, 2011) como para las primeras dinastías (Gandonnière, 2014). Esta breve introducción da cuenta del aumento en los estudios sobre la cacería en la historia temprana de Egipto, así como de la evidencia acerca de la cacería del hipopótamo, lo cual hace posible y hasta necesaria la discusión (basándonos en la nueva evidencia) de sus diversas interpretaciones. 


\section{Nuevos descubrimientos...}

Los excavadores del sitio de Hierakonpolis conmovieron el mundo académico en 2009 con un sorprendente anuncio. Algunos animales desenterrados en ese sitio mostraban fracturas y patologías compatibles con un cautiverio más o menos prolongada (Linseele et al., 2007; 2008; van Neer y Linseele, 2009). La noticia no se hizo esperar: ¡habían encontrado el primer zoológico de la historia! El Archaeological Institute of America lo colocó entre los “mejores diez descubrimientos de 2009”, y la noticia logró gran difusión y notoriedad.

Más allá de la finalidad eminentemente propagandística de utilizar la palabra zoológico, objetivamente se trata de un importantísimo descubrimiento.

Ya Barbara Adams había descubierto el complejo que rodea la Tumba 16 en Hierakonpolis, dentro del cual se hallan varias tumbas de gran tamaño con enterramientos humanos asociados a algunos animales. Si bien algunos de los restos animales se identifican como ofrendas rituales de alimento (van Neer et al., 2011: 165) otros llaman la atención por haber sido enterrados por entero, es decir, sin ser descuartizados antes (van Neer et al., 2004). Existe también una serie de diez tumbas en las que se han enterrado veintiocho animales (van Neer et al., 2011: 175) sin estar asociados a enterramientos humanos, incluyendo ejemplares exóticos como el elefante africano (Loxodonta Africana) y el uro (Bos Primigenius), ambos mamíferos de gran tamaño y que debieron haber cumplido un rol importante en la economía simbólica del poblado predinástico (van Neer et al., 2011: 186).

Otros mamíferos enterrados incluyen mandriles (Papio Anubis), un leopardo (Panthera Pardus), un gato salvaje (Felis Chaus), un búfalo (Alcelaphus Buselaphus); pero el hallazgo más relevante para este artículo es la presencia en dicho complejo de tumbas de un joven ejemplar de hipopótamo (Hippopotamus Amphibius), el cual como otros de los animales nombrados (van Neer et al., 2015: 1) presenta patologías óseas compatibles con un cautiverio más o menos prolongado, lo cual parece contradecir el consenso de la comunidad de egiptólogos que parece aceptar en conjunto (con la excepción de los dos autores citados más arriba) que la muerte de este peligroso animal era producida en el sitio de la cacería.

Así describen el hallazgo Linseele y van Neer:

To our surprise we found a healed fracture on the lower part of the fibula (...) proving that this young hippo indeed spent a rather long time under human control. The location of this fracture is typical of animals that are constrained by a rope tied around their lower hind leg and break their own bone while struggling to be free (2009: 12).

¿Se trata de un caso aislado o puede este nuevo hallazgo obligarnos a impugnar la opinión académica general acerca de la cacería del hipopótamo? El joven espécimen descrito por Linseele y van Neer fue hallado en la Tumba 32 de la localidad conocida como cementerio de élite HK6, asociado a los restos de un hombre enterrado junto con perros. También fueron hallados algunos tiestos muy pequeños, que los arqueólogos del sitio identificaron como los "restos destrozados de objetos modelados en yeso y decorados con motivos en pintura roja, negra y blanca” (Pieri y Friedman, 2010: 14), es decir lo que pudo haber sido un modelo de equipo de cacería, aunque las piezas son demasiado minúsculas para que tal identificación sea inequívoca. Basándose en esta evidencia, y teniendo en cuenta que los perros muchas veces eran un símbolo de la cacería de animales salvajes (Hendrickx, 2006; 2011), los excavadores propusieron que tal tumba hubo pertenecido a un cazador de hipopótamos.

El perro era, de hecho, el único animal doméstico en aparecer con frecuencia en la cerámica White CrossLined de Nagada I (Hendrickx, 2011: 110), y esto debido a que era un símbolo no de los households predinásticos sino de la caza, esto es, la lucha del cazador contra las fuerzas del caos encarnadas en los animales salvajes. Sin embargo, esta proposición sólo vale en lo que respecta a la cacería en el desierto. Los 
perros no tenían ninguna participación en la caza en el ámbito del río Nilo. En estos casos la iconografía del período los reemplaza por botes (Zajac, 2008; Hendrickx, 2013).

Sólo conozco tres ocasiones (Graff, 2009) en que coexisten en la misma representación el perro y el hipopótamo: El plato CG2076, actualmente en el Museo de El Cairo (de Morgan, 1896: Tf. II, No. 5; Hartmann, 2008) y dos vasos altos, el primero encontrado en la Tumba U-415 (Dreyer et al., 2003: 83 Abb. 6a) y otro artefacto que actualmente se encuentra en el Oriental Institute de Chicago (Figura 1; Cat. No. OIM E 8923. Ayrton y Loat, 1911: Pl. 27.12). Los tres fueron hallados en el sitio predinástico de Abydos y están datados en Nagada I.

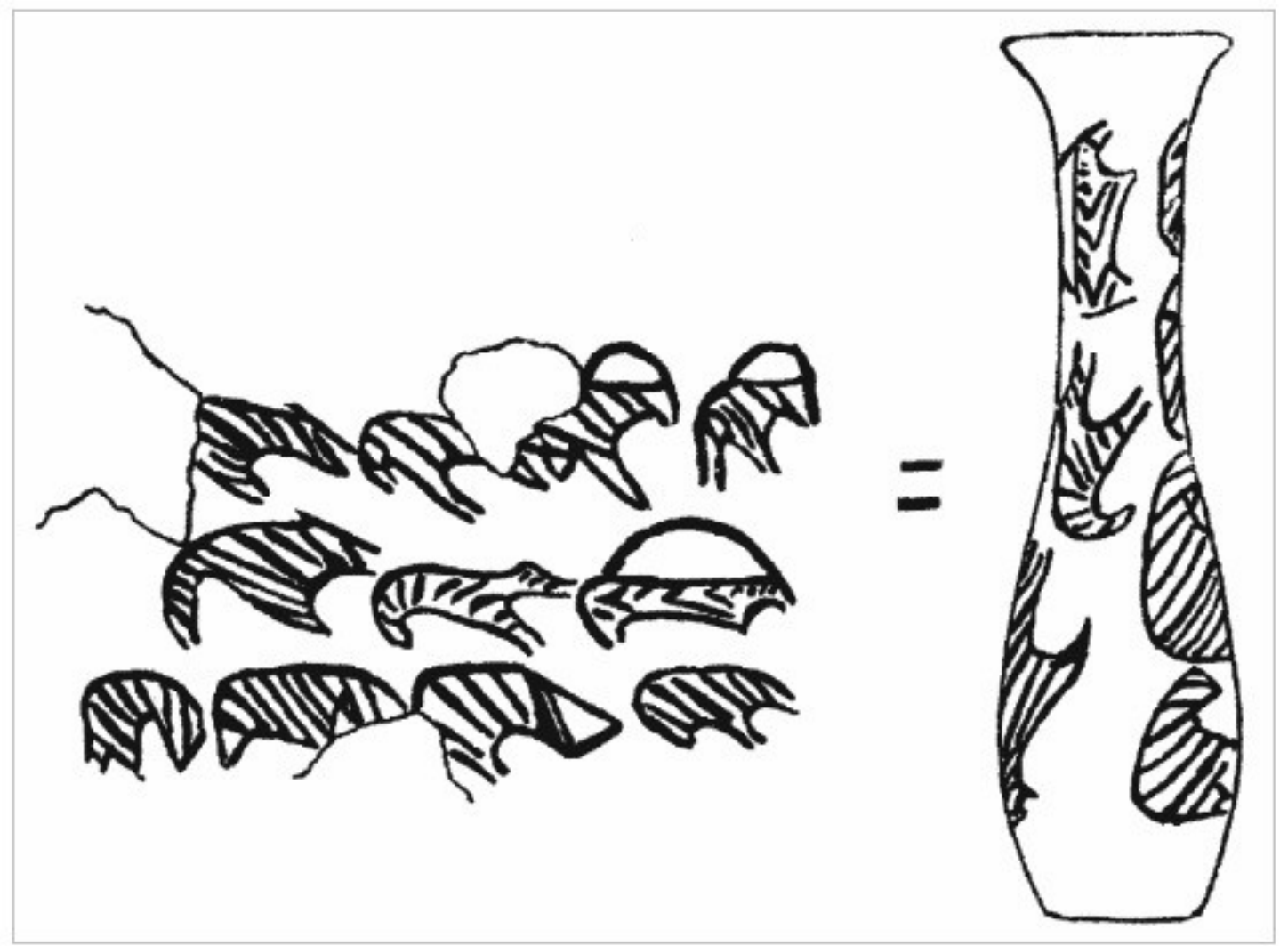

Figura 1. Vasija del Oriental Institute, Cat. No. OIM E 8923 (Ayrton y Loat, 1911: Pl. 27.12)

En cuanto al vaso OIM E 8923, Emily Teeter señala:

The position of the dogs is significant. In the two upper rows with desert animals, they clearly refer to hunting with dogs, but dogs have no part in hippopotamus hunting. In whatever order the rows are considered, the dog preceding the hippopotami will always follow a row ending with another dog, suggesting that the row with hippopotami is also a reference to hunting (Teeter, 2011:154).

Los perros y los hipopótamos están, como podemos ver, ligeramente conectados en relación con las actividades cazadoras. No es impensable, sin embargo, que en estas comunidades fuera el poseedor de un gran prestigio aquel personaje que pudiera controlar animales salvajes tanto en el Nilo como en el desierto. El individuo enterrado en la Tumba 32 pudo haber sido el detentador de ese tipo de estatus en el Hierakonpolis predinástico.

Por otro lado, ciertos petroglifos hallados en el desierto oriental (Hendrickx et al., 2009: 217; Hendrickx, 2011: 120) muestran figuras humanas con tocados de plumas de avestruz, colas de cánidos y atavíos (o 
pintura corporal, Cf. Baduel, 2008) con complicados dibujos, dos de ellos con hipopótamos dibujados o bordados (Figura 2). Gracias a estas representaciones, los cazadores reúnen en sí el control sobre los animales del Nilo y del desierto (simbolizado en las plumas de avestruz y en la cola de cánido, observable en otras representaciones similares), "encarnando la dualidad de su mundo" (Hendrickx et al., 2009: 218). Según estos autores, los grupos de cazadores predinásticos entendían su actividad como la reconciliación del ámbito nilótico con el Sahara y simbólicamente lo expresaban en la iconografía. Los cazadores obrarían de esta forma como bisagra entre biomas diversos (Darnell, 2009: 88).

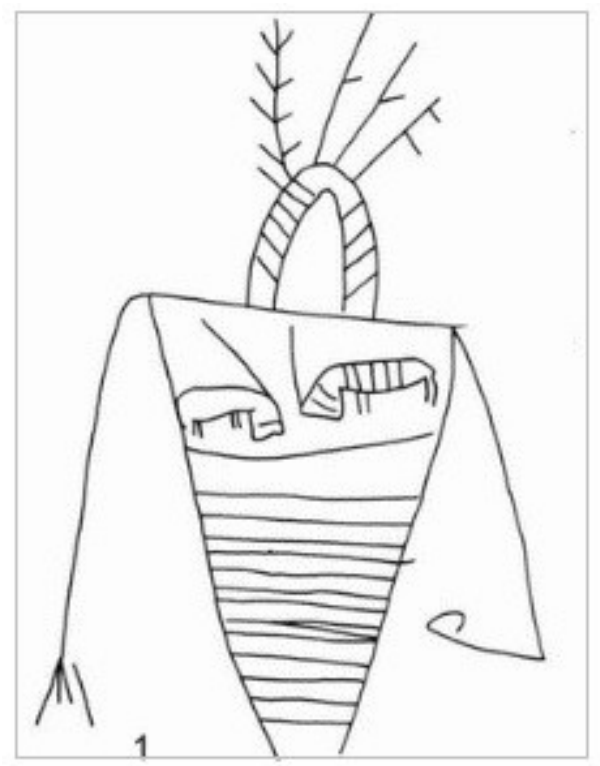

Figura 2. Petroglifo de Was Ha Waset, Cat. No. WHW 90 (Hendrickx et al., 2009: 216 fig. 23.1)

Otros enterramientos de animales encontrados en el cementerio de élite HK6 incluyen ciento diez animales domésticos y treinta y ocho salvajes pertenecientes a doce especies distintas (van Neer et al., 2015: 1). De acuerdo a los zooarqueólogos, por lo menos una veintena de estos animales fueron mantenidos en cautiverio durante cierto tiempo antes de su muerte, a juzgar por las patologías observadas en sus huesos (fracturas defensivas y producidas tras ser atados por un tiempo prolongado). Estos incluían, como vimos más arriba, algunos mandriles, un elefante, un leopardo, cocodrilos, un uro, y por supuesto hipopótamos (van Neer et al., 2015: 18). Diane Flores (2004) ya había señalado la naturaleza única del cementerio HK6 en cuanto al número de enterramientos animales, y de acuerdo a Reneé Friedman

the wide range of animals may have symbolically provided protection against the natural chaos they represented, the captivity and eventual slaughter of these animals a way in which chaos was brought under control (...) The healed injuries observed on some of these animals indicate that they were held in captivity for a minimum of four to six weeks. The creation and maintenance of royal menageries is known to have been a means of legitimising the rule of New Kingdom pharaohs, and it may have served this purpose already at this early time (Friedman et al., 2011: 186).

Como queda claro a partir de esta cita, el cautiverio de animales salvajes y peligrosos efectivamente tenía lugar en Hierakonpolis, y probablemente sirviera el propósito de otorgar legitimidad a los líderes de la comunidad. El liderazgo en estas sociedades estaba basado en la habilidad para controlar el caos y mantener el orden en la comunidad, y el control sobre los animales salvajes era uno de los medios para conseguirlo (Maydana, 2015), tal y como es posible observar en algunos artefactos predinásticos como el mango de cuchillo de Gebel el-Arak y numerosas paletas cosméticas (Köhler, 2002; Hartung, 2010; Hendrickx, 2013). 
Los líderes predinásticos eran identificados en su iconografía por dos características específicas: estaban fuertemente asociados a lo ritual y a la violencia en todas sus formas (Campagno, 2016: 19). Eran representados junto con armas, o en combate contra enemigos humanos o animales salvajes (Gayubas, 2016: 35). Por supuesto, no podemos dejar de lado el aspecto económico del liderazgo, ya señalado por Hoffman (1989) pero invisible en la iconografía del período. La cacería de animales salvajes (o por lo menos su representación) cumplía entonces un rol importante en los procesos de creación y mantenimiento de jerarquías de estas comunidades preestatales.

\section{3. ...y viejas hipótesis}

En la iconografía predinástica nunca aparecen animales muertos $\underline{1}$, y sólo en algunos casos se representaba el momento de su cacería (Hendrickx, 2011: 121; 2013: 249). Es entendible entonces que haya pervivido en los estudios sobre este período la idea que los animales no eran muertos durante la expedición de caza sino capturados vivos. Fue propuesta por primera vez por Robert Mond y Oliver Myers en la década de 1930, aunque sin suscitar demasiado interés por parte de los académicos. Estos autores se mostraron interesados en ciertas cerámicas decoradas que mostraban interacciones entre hipopótamos y seres humanos. En particular se refirieron a un tazón (Figura 3, Metropolitan Museum No. 12.182.15) que fuera publicado por primera vez por Myers (1933: 55) y que muestra dos hipopótamos y un hombre. Ambos animales poseen unas líneas onduladas que se proyectan a partir de sus fosas nasales, en el primero ascendiendo hasta el borde mismo del tazón y en el segundo hipopótamo terminando en las manos del hombre. Esta pieza es interesante porque se trata claramente de un artefacto de élite (la mayoría de los recipientes decorados lo son), a pesar de provenir de Naga ed-Deir, un pequeño cementerio cercano a Armant y que, como explica Kathryn Bard, era considerado demasiado pequeño para generar diferenciaciones sociales: "an elite class of society would have emerged at large centres like Nagada, not in small farming villages such as Armant” (Bard, 1988: 54, Cf. Griswold, 1992).

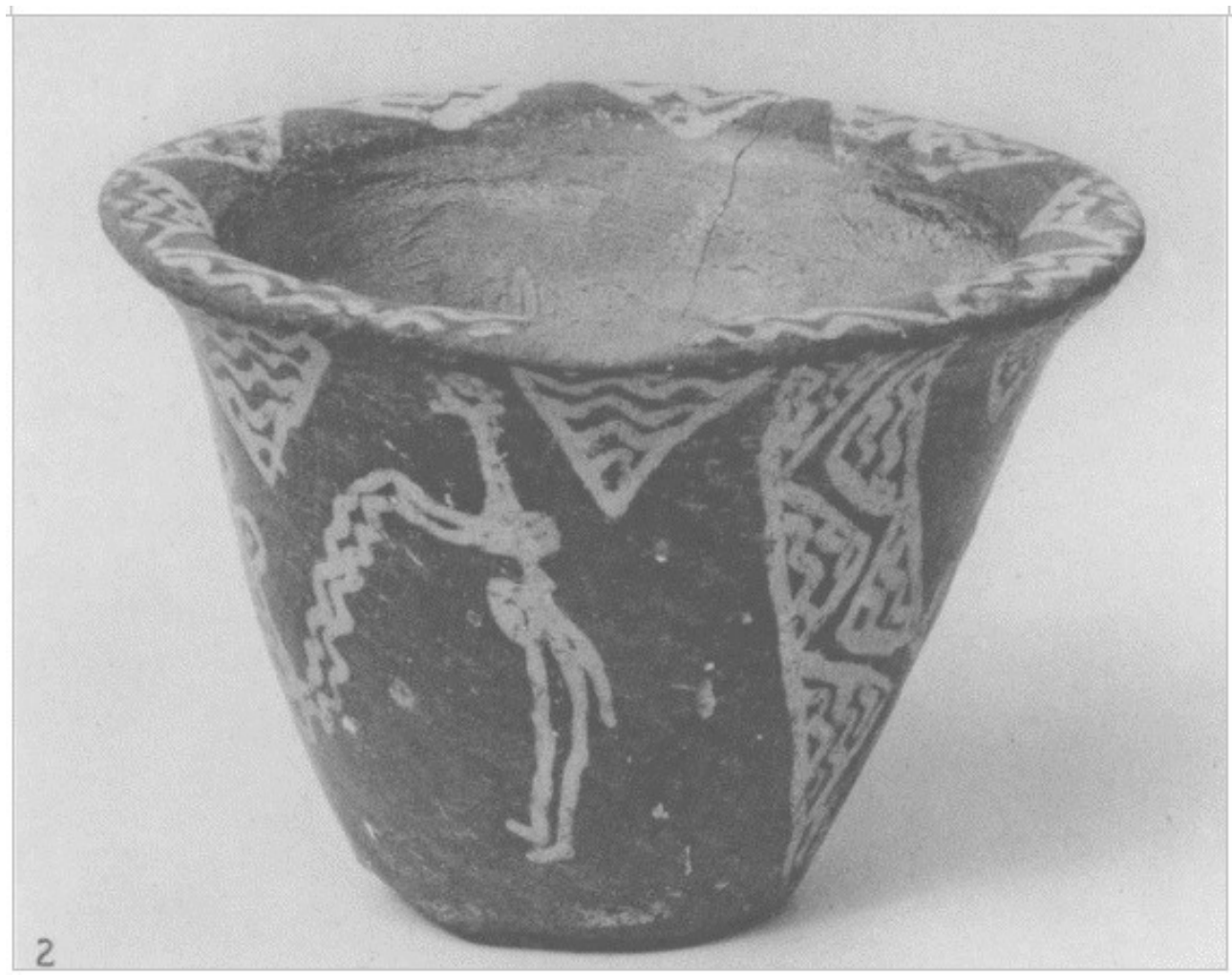

Figura 3. Tazón del Metropolitan Museum, Cat. No. 12.182.15 (Myers, 1933: 55 Pl. XI.2) 
A partir de esta evidencia y haciendo despliegue de viva imaginación Myers propone una serie de posibles interpretaciones:

The impression given is that of vapour rising from the nostrils of the animal and being caught by the man, but, even allowing for a magical interpretation, this seems to be an unlikely hypothesis. It might represent a man feeding the hippopotami, but in that case there is no purpose in the wavy lines ascending from the second beast. There is another possible explanation; that the ovals projecting from the animals' noses may not be nostrils (...) but rings, and that the wavy lines are ropes by which the hippopotami are being led (Myers, 1933: 55)

De las tres posibilidades descritas en aquella oportunidad, Myers terminó por convencerse de que la última era la más plausible, y como tal la desarrolla algunos años después (Mond y Myers, 1937: 38-42). En esta oportunidad los autores plantean una hipótesis más elaborada en relación a la guía de los hipopótamos. Ellos creían que las líneas onduladas representaban una suerte de bridas que permitían a los egipcios predinásticos utilizar a los hipopótamos (domesticados) para espantar a los cocodrilos de las áreas de pesca. Por supuesto, el problema central de esta hipótesis es que nunca fueron hallados dispositivos de este tipo en el registro arqueológico, pero hay un argumento aún más contundente en contra de la teoría de Mond y Myers, y este es el comportamiento naturalmente agresivo del hipopótamo, que vuelve al animal virtualmente imposible de ser domesticado (Manlius, 2000; Kingdon y Hoffman, 2013).

Tal teoría no encontró reconocimiento ni fue retomada por otros egiptólogos debido a que era muy poco realista. Sin embargo, una egiptóloga alemana descubrió décadas más tarde que podía después de todo no ser completamente desacertada. Parte importante del trabajo doctoral de Almuth Behrmann (1996) está dedicada a proponer una nueva lectura de la evidencia iconográfica de la cacería del hipopótamo. En su óptica muchas representaciones de la cacería del hipopótamo muestran en realidad la captura del animal y su posterior cautiverio, y los argumentos que utiliza para defender esta postura pueden llegar a ser más que convincentes.

Para empezar, la autora retoma la polémica acerca de las líneas onduladas que aparecen en muchas representaciones saliendo del cuerpo de los hipopótamos y la mayor parte de las veces terminando en las manos de los cazadores. Se muestra suspicaz respecto de este comportamiento, ya que los hombres sosteniendo a los animales no parecen estar realizando un gran esfuerzo, que se manifestaría en una cuerda tensa, recta. Esto se explica desde su punto de vista porque se trata en realidad de la representación de un animal domesticado, dócil. Luego se concentra en algunos elementos de la decoración de cerámicas que podría interpretarse en el mismo sentido. Por ejemplo, las líneas en zigzag comúnmente entendidas como representativas del agua o como marca de un ambiente acuático podrían en realidad ser "redes de caza" (Behrmann, 1996: 135). Además, marcas geométricas y rectangulares como aquellas presentes en el recipiente 12.182.15 podrían ser vistas como "estanques" o “corrales" donde los animales eran retenidos (Behrmann, 1996: 135). En cuanto a las líneas entrecruzadas que rellenan el cuerpo de varios hipopótamos, en opinión de Behrmann representarían redes. También opina que las marcas en el cuello presentes en algunos pendientes con forma de hipopótamo serían collares (Behrmann, 1996: 138).

Behrmann concluye que lo que la iconografía realmente mostraba era que los hipopótamos no eran muertos en el sitio de caza sino apresados con vida. Como en el caso de Mond y Myers, subsiste la cuestión que su interpretación de la iconografía no se corresponde con el registro arqueológico. Por lo menos esto fue así hasta hace muy poco. Seguramente la Dra. Behrmann estaría satisfecha con el reporte de excavación de 2009 en Hierakonpolis. Se trata sin ir más lejos de la evidencia más sólida hasta el momento para sostener su teoría.

Quisiera aprovechar la oportunidad para aportar otro tipo de evidencia, de carácter simbólico, que podría hacernos descartar la idea de la muerte del hipopótamo durante la cacería. Säve-Söderbergh (1953: 15-17) 
señaló que desde las representaciones más tempranas se mostraba al rey como cazador de hipopótamos, en el rol de un joven y vigoroso héroe, guardián del orden cósmico y destructor del caos que emerge desde el agua. "The struggle of the King against the hippopotamus symbolized the struggle with and victory over the powers of chaos (...) which victory implied the creation of a new world” (Säve-Söderbergh, 1953: 15). Esta victoria es simbólica y se encuentra reflejada en incontables pinturas, grabados y textos a lo largo de toda la historia egipcia.

Por ejemplo, en De Isis y Osiris Plutarco se refiere a Seth / Tifón de la siguiente manera: "ellos [los egipcios], de los animales domésticos, le asignan el más estúpido, el asno, y entre las fieras, las más salvajes, el cocodrilo y el hipopótamo” (Isis y Osiris, 50).

La identificación del dios Seth con el hipopótamo está bien atestiguada desde el Reino Antiguo (Behrmann, 1996: 67-77). Seth era el asesino de Osiris, enemigo del faraón, dios de la confusión (Te Velde, 1967) y eterno rival de Horus, el dios halcón. Pero cuando Horus, hijo de Osiris, finalmente fue capaz de capturar al asesino de su padre

Tifón fue vencido [por Horus], pero no aniquilado. Pues la diosa señora de la tierra no permitió que fuera aniquilada por completo la naturaleza antagonista de la humedad, sino que lo puso en libertad y lo dejó ir, con el propósito de que se mantuviera la mezcla, pues no era posible que el cosmos fuera perfecto faltando y desapareciendo el elemento ígneo. (40, 367 A).

Además de que sería irresponsable trasladar este relato de obvia influencia pitagórica y de confección tardía a los tiempos predinásticos, tampoco existe ninguna evidencia que avale una hipótesis parecida. Sin embargo, como ejercicio de pensamiento configura un interesante ejemplo de un principio que podría sostener la tesis de la captura del hipopótamo: sólo se puede mantener el orden mientras haya una amenaza a tal orden, de forma que el caos debe ser mantenido lejos pero nunca destruido enteramente, so pena de romper el balance cósmico entre orden y caos. Y, como hemos visto, la responsabilidad de sostener ese balance cósmico era prerrogativa del líder.

En lo que queda del artículo me gustaría participar de la discusión desde otro ángulo, además de volver a evaluar la evidencia disponible y formular una propuesta al respecto.

\section{Reevaluando la evidencia: argumentos arqueo-históricos}

Sabemos gracias a la paleontología que el hipopótamo fue cazado para obtener alimento por el género homo desde 2.000.000 BP (Hill, 1983), y en el valle del Nilo desde el Pleistoceno superior hasta el Holoceno temprano (45.000 - 15.000 a.C.) (Marks, 1968: 315). Se han hallado en el Fayum esqueletos de hipopótamo y elefante con puntas de proyectil incrustadas que datan de tiempos anteriores a Nagada (Hayes, 1964: 220) ${ }^{2}$. Sin embargo, con el desarrollo posterior de la agricultura el porcentaje de proteína animal obtenida mediante la cacería se desploma significativamente en la dieta predinástica (Abd el Karem, 2013). No obstante, los hipopótamos continuaron siendo cazados hasta su extinción de Egipto en el s. XIX (Manlius, 2000; 2006: 105).

En las sociedades neolitizadas del predinástico, entonces, la proteína de animales salvajes se volvió claramente marginal en la dieta de sus habitantes (Linseele y van Neer, 2009:124), pero esto contradice la profusión de escenas de cacería que aparecen en el corpus de representaciones predinásticas (Altenmüller, 1980: 224). En efecto, las escenas de cacería (junto con las procesiones de botes) son absolutamente dominantes en el registro iconográfico nagadiense (Graff, 2009; Hendrickx, 2011), lo cual contrasta en gran medida con el despreciable lugar que la cacería real ocupó en las costumbres predinásticas. 
La evidencia arqueozoológica es categórica en este aspecto. Como señalan Linseele y van Neer (2009), el número de huesos de animales salvajes hallados en Hierakonpolis era inusualmente alto para un asentamiento predinástico, y ello puede ser explicado por la naturaleza especial de este sitio, que ha sido llamado “complejo templo-taller" (Holmes, 1992: 37). Este sitio del Alto Egipto era efectivamente un importante centro religioso, lo cual probablemente haya contribuido a su crecimiento durante las últimas fases del predinástico (Adams, 1995: 58). La alta concentración de restos de animales salvajes en Hierakonpolis es una anomalía, explicada en parte por la naturaleza ceremonial del sitio, y como tal exceptio probat regulam.

En base a lo antedicho es posible argumentar que la cacería durante el período de Nagada era sobre todo un motivo iconográfico. La cacería efectiva tenía lugar, pero no tan seguido como la iconografía parecería indicar. El porqué de esto apunta a la eficacia simbólica que pudo llegar a tener tal motivo, que ilustraba el control de las tumultuosas fuerzas del animal salvaje por parte de figuras de liderazgo y el papel de estos cazadores como vínculos entre espacios diversos. Es en este sentido que Darnell propone interpretar ciertos petroglifos del Desierto Occidental cerca de Tebas (ver Figura 2) como ejemplos de lo que llama la nilotización del desierto. Según él, este tipo de figuraciones (se reportaron más de quinientas inscripciones de diversos períodos) "makes of the background the waters of the Nile, thereby transforming the gebel medium into a simulacrum of the Nile” (Darnell, 2009: 88). Se trataría en efecto de traer a las zonas áridas la imagen del río y con él de la inundación que da vida (Darnell, 2002: 111).

La falta de representaciones de la muerte de los animales sumada a las evidencias de que la cacería no cumplía un rol alimentario real hizo en los últimos años que algunos egiptólogos retomen la antigua tesis acerca de que en las representaciones aparece la captura de animales, y no su muerte. Según Hendrickx (2011: 121-123) la captura de los animales tendría una ventaja sobre su matanza: la mayor facilidad del transporte de vuelta a la aldea. La frecuente aparición de lazos, sogas y trampas y la actitud de los cazadores representados parece sugerir esto. Sin embargo, el autor se apresura a aclarar que existen dos excepciones a su regla: el hipopótamo y el asno salvaje (ver nota 1). Para probar la excepción en el caso de las escenas de cacería del hipopótamo alega una continuidad entre estas y las de época dinástica en que el rey claramente arponea al hipopótamo. Vera Müller (2008) estudió ciertas escenas en que se asimila la caza del hipopótamo a algunas imágenes de enemigos decapitados, lo cual las emparenta con el "prototipo real" (SäveSöderbergh, 1953). De hecho, se conservan una serie de etiquetas de marfil y cilindros sello del reinado de Den (Dinastía I) en los cuales se observa al rey abatiendo al hipopótamo, tanto por medio de lanzas como en combate cuerpo a cuerpo (Roche, 2014: 77), lo cual puede hacer pensar en una continuidad de la práctica de cacería del hipopótamo entre el predinástico y el período tinita (Roche, 2014: 84). Sin embargo, esta hipótesis tiende a dejar de lado que entre ambos períodos se produjo un cambio sociopolítico enorme y de profundas implicaciones: el surgimiento del Estado (Campagno, 2002). En otro trabajo he propuesto (2017: 52) tener en cuenta que la sociedad predinástica era fundamentalmente distinta a la de la Dinastía I en que la lógica de organización social dominante es aquella en que un personaje o grupo detenta el monopolio de la coerción legítima. La división social que de ello resulta tiene ciertos mecanismos que legitiman la posición social del grupo dominante, entre ellos la habilidad para cazar al hipopótamo (Müller, 2008; Hartung, 2010; Hendrickx, 2011). Sin embargo, la sociedad predinástica estaba organizada sobre la base de un entramado social que respondía a los mecanismos del parentesco, por lo que la existencia de un grupo social que poseyese el monopolio de la violencia legítima era impensable. En este sentido, la representación de la cacería del hipopótamo tenía un rol muy distinto en el proceso de construcción de liderazgos de las comunidades del Alto Egipto. La discontinuidad se observa en el registro arqueológico en que, por ejemplo, la mayoría de las representaciones de cacería del hipopótamo en cerámicas pertenecen a Nagada I-IIa (Graff, 2009: 60) mientras que hacia el fin del predinástico y el inicio de la Dinastía I no se observa este tipo de figuraciones. En cambio, los motivos que dominan el registro iconográfico son la "planta de Nagada" 
(Hendrickx y Eyckerman, 2008, 2012) y cierto tipo de bote específico del período (Lankester, 2012: 47).

Volviendo a la cuestión de si el hipopótamo era muerto en la cacería o capturado vivo, un argumento convincente a favor de lo primero lo da el propio Hendrickx en un artículo reciente, al afirmar que "capturing a mature hippopotamus would have been a very difficult undertaking” (Hendrickx, 2013: 249). En efecto, el criterio práctico es el más útil aquí. La etnografía resulta ilustrativa en este sentido.

\section{Reevaluando la evidencia: argumentos etnográficos}

En su ya clásico ensayo acerca de la cacería del hipopótamo, el egiptólogo sueco Torgny Säve-Söderbergh acertadamente señala que los arpones representados en las tumbas del Imperio Nuevo son muy similares a aquellos utilizados en la actualidad por los shilluk de Sudán y los Wandamba de Tanzania (1953: 8). El método que utilizan para cazar hipopótamos es agitar el agua alrededor de sus botes con un largo palo hasta que la bestia emerge del río, para entonces atacarla con los arpones buscando siempre las partes más blandas de modo que la punta penetre en la gruesa piel del animal hasta la carne. Intentan así alcanzar las áreas más vulnerables del animal, las fosas nasales, los costados y el interior de su boca (lo cual impide a su vez que el animal se sumerja nuevamente). La cabeza del arpón está diseñada de forma que no se pueda desprender del animal una vez adentro. Este está atado a una larga cuerda que a su vez posee en el otro extremo unos dispositivos flotantes que ayudan a los hombres a ubicar perfectamente a la presa sumergida para poder seguir arponeándola hasta matarla (Gregorius, 1964: 204).

Existen incluso grabaciones en video de la cacería de los hipopótamos en las marismas (Rouch, 1950). En el documental de Jean Rouch el espectador pasa de ver la fiereza de la lucha entre los cazadores y la bestia, y un corte lo traslada al plano de un hombre joven nadando y jugando con una cría de hipopótamo que tiene su pata trasera atada a un árbol, en la misma forma que lo estuvo en otro tiempo el cautivo hipopótamo de Hierakonpolis (Figura 4).

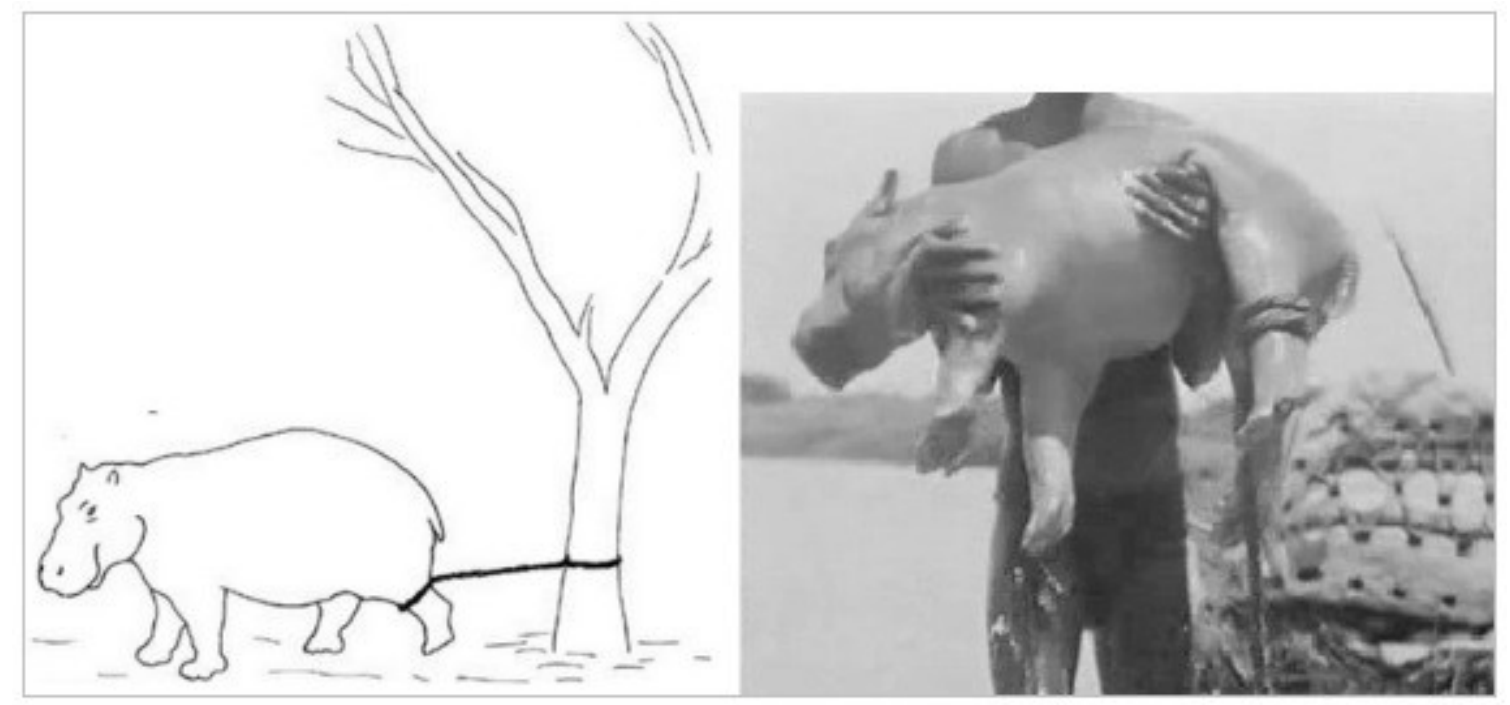

Figura 4. Comparación entre la reconstrucción realizada por van Neer y Linseele (2009: 12) y un fotograma del documental de Jean Rouch Bataille sur le Grand Fleuve (1950).

Otro etnógrafo provee un diagrama de los arpones (Culwick, 1932: 277), y cuando comparamos a estos con algunos dibujos de armas en cerámicas tal y como podemos ver en los fragmentos de una vasija predinástica (AM 1924.326, Brunton y Caton-Thompson, 1928: Pl. XLVIII.3) notamos un gran parecido. Otra vasija teriomorfa en el Ashmolean Museum (E 2802, Petrie, 1901: Pl. XIV f. 67) contiene representaciones del mismo arpón (Figura 5), y otra arma similar está presente en el costado de un vaso alto decorado de Abydos 
(de la Tumba U-637/3, Hartmann, 2003: 83) en conexión con un hipopótamo haciendo clara referencia a la cacería de este animal.

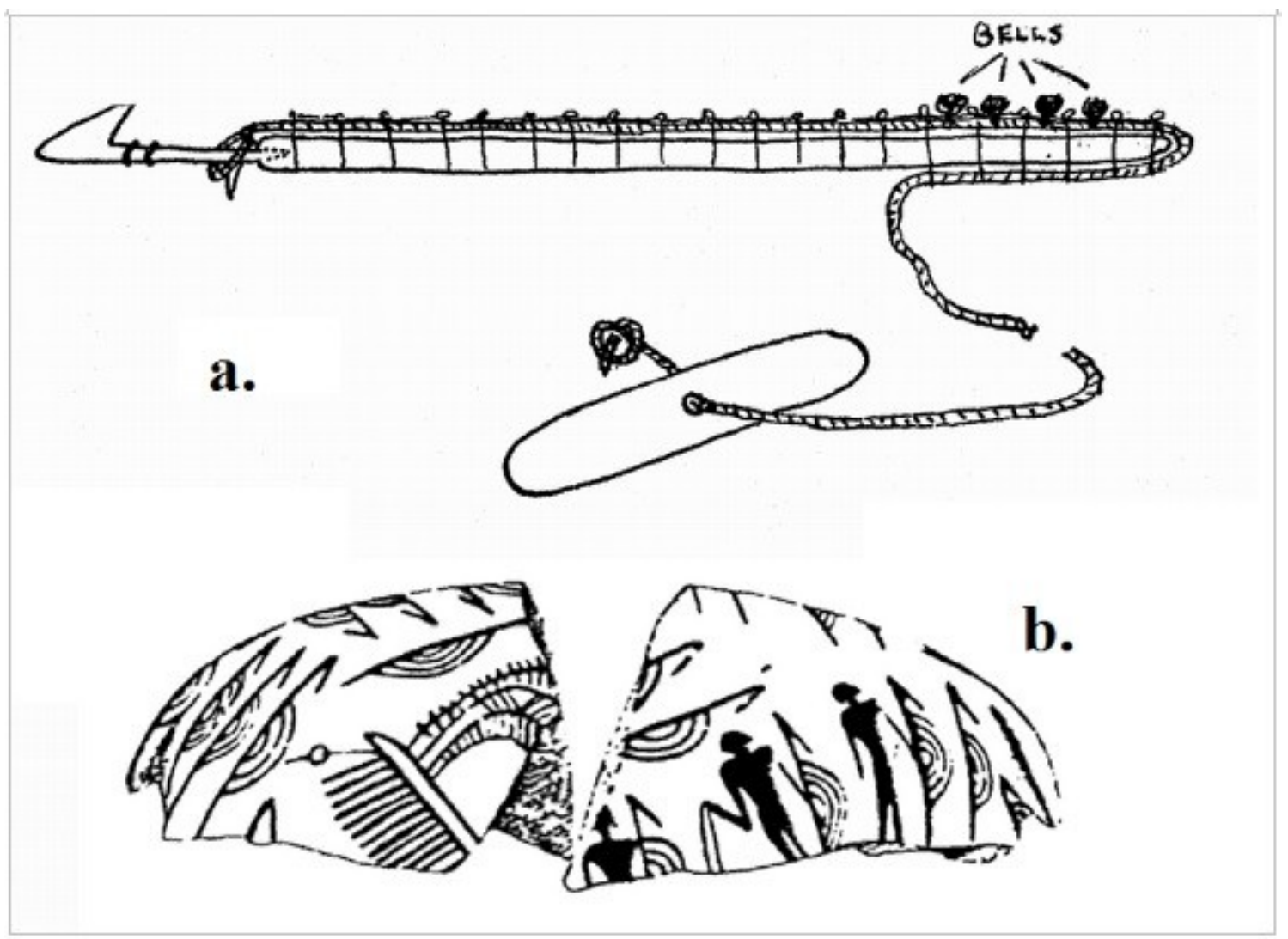

Figura 5. Comparación de instrumentos de épocas distintas:

a. Dibujo de un aparejo para cazar hipopótamos (Culwick, 1932: 277)

b. Fragmentos de un recipiente en forma de hipopótamo con decoración alusiva a la cacería del mismo animal (Behrmann 1989: Dok. 40)

Dispositivos circulares que parecen ser flotadores pueden verse en petroglifos localizados en Wadi Abu Mu Awwad (Morrow y Morrow, 2002: 109) y en Wadi Hammamat (Winkler, 1938: Fig. 8). Ejemplos cerámicos incluyen un plato en el Hearst Museum (Cat. No. 6-3559, Lythgoe y Dunham, 1965), un plato de Mostagedda (Cairo Museum, JE 52835), un vaso decorado probablemente proveniente de Gebelein (Berlin Museum Cat. No. 23222, Scharff, 1931: 117), el plato CG 2071 del Museo de El Cairo, un recipiente decorado hallado en la Tumba U-637 en Abydos, y un plato decorado del Ashmolean Museum (AM 1909.1026).

Las líneas que Myers malinterpretó como vapor o riendas son claramente las cuerdas que se encuentran adheridas a la cabeza del arpón que está fijo a su vez en la carne del animal. Es normal que salgan sobre todo de las fosas nasales, al tratarse de la parte menos resistente del animal y donde los cazadores buscan atacar. En cuanto a la aparente falta de tensión, se trata de un procedimiento adoptado corrientemente en las representaciones egipcias que consiste en omitir los elementos de tensión enfatizando en cambio la facilidad con que las figuras de poder controlan la situación. Esta es una estrategia ya observada por Säve-Söderbergh en los murales egipcios del Reino Nuevo, cuando por ejemplo se pintaba a la mujer del difunto plácidamente sentada en el mismo bote en el que su cónyuge cazaba al hipopótamo (Säve-Söderbergh, 1953: 8). Gracias a ello, podemos pensar que la apariencia relajada de las personas que sostienen mediante cuerdas a los animales no es más que una exhibición de poder, con el objetivo de "dar la idea de calma a la escena, y remarcar la absoluta superioridad del imperturbable poder sobre el fiero y peligroso animal” (SäveSöderbergh, 1953: 10). Las representaciones ilustran al cazador como un ser tan poderoso que puede sostener 
al animal (que puede llegar a pesar tres mil kilogramos) con sus manos desnudas y sin ayuda alguna.

En cuanto a las líneas cruzadas que cubren el cuerpo de los hipopótamos en algunas representaciones, estas no son más que la característica central de la llamada White Cross-lined pottery, como Petrie la llamó precisamente porque sus figuras estaban comúnmente rellenas con líneas entrecruzadas. La mayoría de los grandes animales en este tipo de cerámica están rellenos con tales líneas. Los rectángulos y líneas en zigzag podrían bien representar varias cosas distintas, incluyendo los estanques y corrales que Behrmann creyó ver, pero cualquier propuesta es tan buena como las demás.

\section{Conclusiones}

La cacería del hipopótamo, tal como evidencia la etnografía moderna y la iconografía antigua, habría sido una práctica en la que se busca la muerte del peligroso animal en el mismo acto. Las tesis contrarias, tanto la de Mond y Myers como la de Behrmann, se apoyan fuertemente en la interpretación de algunos elementos de la iconografía, dejando de lado otros que no cooperan con sus teorías.

En el presente artículo presenté dos conjuntos de hipótesis acerca de las representaciones de la cacería del hipopótamo durante el predinástico: un conjunto que podríamos llamar consensualista ya que busca interpretar las relaciones entre el humano y el animal en términos de convivencia pacífica entre ambos mundos (los animales eran conducidos a la aldea, para cohabitar el mismo espacio que el hombre); y otro conjunto que hace hincapié en la relación violenta entre el hipopótamo y el cazador, que identifica su cacería con el intento de matar al animal.

El primer conjunto de propuestas tiene en la egiptología una expresión acabada en el influyente Bestiaire Égyptien de Philippe Germond y Jacques Livet (2001) y entiende al mundo animal como complementario del humano, como dos mitades de una esfera que abarca al cosmos. De esta manera, hombre y animal en mutua interdependencia se respetan y cuidan manteniendo un equilibrio cósmico. Según ellos la cosmología egipcia se diferenciaría de las demás por otorgar el mismo status al animal que al hombre; en otras palabras, el demiurgo habría creado un mundo carente de jerarquías definidas, y por lo tanto el hombre (creado a imagen y semejanza de Dios según la tradición occidental de Génesis 1:27) no estaría en un rango superior al animal, por lo que era entendido en términos de paridad.

Por otro lado, la egiptóloga australiana Linda Evans ha dedicado parte de su tesis doctoral a criticar la postura clásica de Germond y Livet. En particular, y tras un extenso estudio de fuentes pictóricas, demuestra que en efecto los egipcios "did not experience a kinship with the animal kingdom, but instead perceived them as distinctly 'other'” (Evans, 2010: 124). En su visión, afín a lo que venimos discutiendo en estas páginas, la relación entre el humano y el animal distaría mucho de ser pacífica y fraternal.

Por supuesto que pretender encasillar la relación que los egipcios tuvieron con los animales a lo largo de tantos siglos en alguno de estos dos extremos sería tan inconcebible como ajeno a mis intenciones. Simplemente, lo que propongo es un cambio en la mirada en que los egiptólogos concebimos esa relación a fin de lograr un mejor conocimiento del período y la sociedad en cuestión.

En conclusión, debe quedar claro que la matanza del hipopótamo está implícita en su cacería, a pesar de no figurarse el animal muerto. Muy probablemente la forma en que los pueblos africanos cazan hoy en día al hipopótamo sea la manera exacta en que los antiguos egipcios lo hacían, y los hombres paleolíticos antes que ellos (Hill, 1983). Todos los etnógrafos coinciden en que el animal era muerto en el río o en la orilla. Es probable por otro lado que ocasionalmente capturaran a las crías huérfanas, como hicieron en Hierakonpolis. La revisión y discusión de estas tesis acerca de la cacería del hipopótamo, a pesar de desmentirlas, es un útil recordatorio de que los egiptólogos no debemos dejar nada por sentado. Muy por el contrario, debemos estar 
preparados en todo momento para cuestionar tanto fuentes como interpretaciones, revisando los problemas desde todos los ángulos posibles.

\section{Notas}

1 Con una notable excepción: el asno salvaje, cuya carga simbólica como representante del caos explica la importancia de neutralizarlo mediante su muerte figurada (Huyge 2009; Hendrickx 2011). De igual manera, en Qurta se halló entre otros petroglifos uno que figura un hipopótamo que presenta rasguños en su cabeza y cuello, lo cual probablemente sea una manera simbólica de cazar al animal (Huyge, 2008: 290; Huyge y Claes, 2009: 26).

$\underline{2}$ Hay razones para pensar que incluso en el VI milenio a.C. el hipopótamo era cazado, no sólo por su carne, sino también con fines rituales: "The long bones and spinal vertebrae of this massive beast (...) are found sometimes bound with sinew and cloth and stuck upright in the ground like columns, evidently as offerings to some divinity or guiding spirit of the chase who seems to have been similarly propitiated in the later prehistoric settlement at Maadi” (Hayes, 1964: 238).

\section{Bibliografía}

Abd el Karem, M. (2013). Die Nutzung tierischer Ressourcen während des 5. Und 4.Jahrtausends v. Chr. in Ägypten. Tesis de maestría inédita. Universität Wien.

Adams, B. (1995). Ancient Nekhen: Garstang in the city of Hierakonpolis. Egypt Studies Association Publication, $n^{\circ}$ 3: SIA Publishing.

Adler, A. (2007). "La guerra y el Estado primitivo”. En M. Abensour (comp.). El espíritu de las leyes salvajes. Una nueva antropología política (pp. 163-168). Buenos Aires: Del Sol.

Altenmüller, H. (1980). “Jagdritual“. En Lexikon der Ägyptologie, Band III (pp. 231-233). Wiesbaden: Otto Harrasowitz.

Ayrton, E. R. y Loat, W. L. S. (1911). The Pre-Dynastic Cemetery at El Mahasna. MEEF 31. Londres.

Baduel, N. (2008). “Tegumentary paint and cosmetic palettes in Predynastic Egypt. Impact of those artefacts on the birth of the monarchy”. En Midant-Reynes et al. (eds.), Egypt at its origins 2. Proceedings on the International conference "origin of the State. Predynastic and early Dynastic Egypt", Toulouse, 5th-8th September 2005 (pp. 1057-1090). Orientalia Lovaniensia Analecta 172. Lovaina: Peeters.

Bard, K. (1988). “A Quantitative Analysis of the Predynastic Burials in Armant Cemetery 1400-1500”. Journal of Egyptian Archaeology, 74, 39-55.

Behrmann, A. (1989). Das Nilpferd in der Vorstellungswelt der Alten Ägypter. Teil I, Katalog. Europäische Hochschulschriften. Reihe XXXVIII, Archäologie, Bd. 22. Frankfurt: Peter Lang.

Behrmann, A. (1996). Das Nilpferd in der Vorstellungswelt der Alten Ägypter. Teil II, Textband. Europäische Hochschulschriften. Reihe XXXVIII, Archäologie, Bd. 62. Frankfurt: Peter Lang.

Brunton, G. y Caton-Thompson, G. (1928). The Badarian Civilization. BSAE \& ERA 46. Londres.

Campagno, M. (2002). De los jefes-parientes a los reyes-dioses. Surgimiento y consolidación del Estado en el antiguo Egipto. Aula Ægyptiaca-Studia 3, Barcelona: Aula Ægyptiaca. 
Campagno, M. (2016). "Surgimiento de lo estatal y liderazgo local en el valle del Nilo (IV-III milenios a.C.)”. En M. Campagno, J. Gallego y C. García Mac Gaw (comps.). Regímenes Políticos en el Mediterráneo Antiguo. Estudios del Mediterráneo Antiguo (pp. 15-30). PEFSCEA Nº 11. Buenos Aires: Miño y Dávila.

Culwick, A. T. (1932). "Hippo hunting amongst the Wandamba of Tanganyika Territory”. Man, Vol. 32, 276278.

Darnell, J. C. (2002). "The Narrow Doors of the Desert”. En David, B. y Wilson, M. (eds.). Inscribed Landscapes (pp. 104-121). Honolulu: University of Hawaii Press.

Darnell, J. C. (2009). “Iconographic Attraction, Iconographic Syntax, and Tableaux of Royal Ritual Power in the Pre- and Proto-Dynastic Rock Inscriptions of the Theban West Desert”. Archéo-Nil N 19, 83-107.

Dreyer, G. et al. (1998). "Umm el-Qaab, Nachuntersuchungen im frühzeitlichen Königsfriedhof, 9./10. Vorbericht”. Mitteilungen des Deutschen Archäologischen Instituts Abteilung Kairo, 54, 77-167.

Dreyer, G. et al. (2009). "Umm el-Qaab, Nachuntersuchungen im frühzeitlichen Königsfriedhof, 13./14./15. Vorbericht”. Mitteilungen des Deutschen Archäologischen Instituts Abteilung Kairo, 65, 43-129.

Evans, L. (2010). Animal Behavior in Egyptian Art. Representations of the Natural World in Memphite Tomb Scenes. Oxford: Aris \& Phillips.

Flores, D. V. (2004). "Funerary sacrifice of animals in the Egyptian Predynastic period”. En S. Hendrickx, R. Friedman, K. Cialowicz y M. Chlodnicki (eds.). Egypt at its Origins. Studies in memory of Barbara Adams. Proceedings of the International Conference 'Origin of the State. Predynastic and Early Dynastic Egypt', Krakow, 28th August - 1st September 2002 (pp. 731-763). Lovaina: Peeters.

Friedman, R. F. (2004). “Elephants at Hierakonpolis”. En S. Hendrickx, R. Friedman, K. Cialowicz y M. Chlodnicki (eds.). Egypt at its Origins. Studies in memory of Barbara Adams. Proceedings of the International Conference 'Origin of the State. Predynastic and Early Dynastic Egypt', Krakow, 28th August - 1st September 2002 (pp. 131-168). Lovaina: Peeters.

Friedman, R. F. et. al. (1999). "Preliminary Report on Field Work at Hierakonpolis: 1996-1998”. Journal of the American Research Center in Egypt, Vol. 36, 1-35.

Friedman, R. F. et al. (2011). “The elite predynastic cemetery at Hierakonpolis: 2009-2010 update”. En R. Friedman y P. Fiske (eds.) Egypt at its Origins 3. Proceedings of the International Conference "Origin of the State. Predynastic and Early Dynastic Egypt”. Londres, 27 de Julio - 1 de Agosto de 2008 (pp. 157-191). Orientalia Lovaniensia Analecta 205. Lovaina: Peeters.

Gandonnière, C. (2014). “Chasseurs et équipes de chasseurs de l’Ancien au Nouvel Empire”. Nehet, Vol. 1. Paris-Sorbonne, 47-69.

Gayubas, A. (2016). “Guerra, territorio y cambio social en el valle del Nilo preestatal”. En M. Campagno, J. Gallego y C. García Mac Gaw (comps.). Regímenes Políticos en el Mediterráneo Antiguo. Estudios del Mediterráneo Antiguo (pp. 31-44). PEFSCEA Nº 11. Buenos Aires: Miño y Dávila.

Germond, Ph. y Livet, J. (2001). Bestiaire Égyptien. Paris: Citadelles \& Mazenod.

Graff, G. (2009). Les peintures sur vases de Naqada I - Naqada II. Nouvelle aproche sémiologique de l'iconographie prédynastique. Lovaina: Leuven University Press.

Gregorius, P. (1964). “Die Wandamba (Tanganyika): Ein Forschungsbericht”. Anthropos, Bd. 59, H. 1./2., 165-217. 
Griswold, W. A. (1992). “Measuring Social Inequality at Armant”. En B. Adams y R. Friedman (eds.). The Followers of Horus. Studies dedicated to Michael Allen Hoffman 1944-1990 (pp. 193-198). ESA 2, Oxbow Monograph 20.

Hartmann, R. (2003). "Keramik”. En G. Dreyer et. al. "Umm el-Qaab. Nachuntersuchungen im frühzeitlichen Königsfriedhof 13./14./15. Vorbericht”. Mitteilungen des Deutschen Archäologischen Instituts Abteilung Kairo 59, 80-85.

Hartmann, R. (2008). "Zwei Fragmente der White Cross-lined Ware aus dem Friedhof U in Abydos zu Gefässen aus dem Ägyptischen Museum Kairo”. En E-M. Engel, V. Müller y U. Hartung. Zeichen aus dem Sand. Streiflichter aus Ägyptens Geschichte zu Ehren von Günther Dreyer (pp. 163-182). Menes 5. Wiesbaden: Otto-Harrasowitz.

Hartung, U. (2010). "Hippopotamus hunters and bureaucrats: Elite Burials at Cemetery U at Abydos”. En F. Raffaele et al. (eds.). Recent Discoveries and Latest Researches in Egyptology: Proceedings of the First Neapolitan Congress of Egyptology, Naples, June 18th - 20th, 2008 (pp. 107-120). Wiesbaden: Otto Harrassowitz.

Hartung, U., Engel, E-M. y Hartmann, R. (2012). “Tell el-Fara'in - Buto 11. Vorbericht”. Mitteilungen des Deutschen Archäologischen Instituts Abteilung Kairo 68, 83-114.

Hayes, W. C. (1964). "Most Ancient Egypt: Chapter III. The Neolithic and Chalcolithic Communities of Northern Egypt”. Journal of Near Eastern Studies, Vol. 23, No. 4, 217-272.

Hendrickx, S. (2006). “The dog, the Lycaon pictus and order over chaos”. En K. Kroeper, M. Chlodnicki y M. Kobusiewicz (eds.). Archaeology of Early Northeastern Africa. In Memory of Lech Krzyżaniak (pp. 723749). Studies in African Archaeology 9. Poznan.

Hendrickx, S. (2011). "L’Iconographie de la chasse dans le contexte social prédynastique”. Archéo-Nil, No. 20, 108-136.

Hendrickx, S. (2013). "Hunting and social complexity in Predynastic Egypt”. Academie Royale des Sciences d'Outre-mer, Bulletin des Séances / Koninklijke Academie voor Overzeese Wetenschappen, Mededelingen der Zittingen, 57, 2-4 (2011), 237-263 [publicado en 2013].

Hendrickx, S. y Eyckerman, M. (2008). "Tusks and Tags: between the hippopotamus and the Naqada Plant”. En Friedman, R. F. y Fiske, P. N. (eds.) Egypt at its Origins 3. Proceedings of the International Conference "Origin of the State. Predynastic and Early Dynastic Egypt". Londres, 27 de Julio - 1 de Agosto de 2008 (pp. 497-570). Orientalia Lovaniensia Analecta 205. Lovaina: Peeters.

Hendrickx, S. et. al. (2009). "Late Predynastic/Early Dynastic rock art scenes of Barbary sheep hunting in Egypt's Western Desert. From capturing wild animals to the women of the 'Acacia House”. En H. Riemer, F. Förster, M. Herb y N. Pöllath (eds.). Desert animals in the eastern Sahara: Status, economic significance and cultural reflection in antiquity. Proceedings of an interdisciplinary ACACIA workshop held at the University of Cologne December 14-15, 2007 (pp. 189-244). Colloquium Africanum 4. Heinrich-BarthInstitut. Cologne.

Herzog, W. (2010). The Cave of Forgotten Dreams. Film documental.

de Heusch, L. (2007[1987]). "La inversión de la deuda (proposiciones acerca de las realezas sagradas africanas)”. En M. Abensour (comp.). El espíritu de las leyes salvajes. Pierre Clastres o una nueva antropología política (pp. 95-120). Buenos Aires: Del Sol.

Hill, A. (1983). "Hippopotamus butchery by homo erectus at Olduvai”. Journal of Archaeological Science 
1983, 10, 135-137.

Hoffmann, M. A. (1989). “Packaged funerals and the rise of Egypt”. Archaeology 42, 48-51.

Holmes, D. (1992). "Chipped Stone-Working craftsmen, Hierakonpolis and the Rise of Civilization in Egypt”. En B. Adams y R. Friedman (eds.). The Followers of Horus. Studies dedicated to Michael Allen Hoffman 1944-1990 (pp. 37-44). ESA 2, Oxbow Monograph 20.

Huyge, D. (2008). “'Lascaux along the Nile': the palaeolithic rock art of Qurta (Upper Egypt)”. Academie Royale des Sciences d'Outre-mer, Bulletin des Séances / Koninklijke Academie voor Overzeese Wetenschappen, Mededelingen der Zittingen, 54,3, 281-296.

Huyge, D. (2009). "Detecting magic in rock art: The case of the ancient Egyptian 'malignant ass'”. En H. Riemer, F. Förster, M. Herb y N. Pöllath (eds.). Desert animals in the eastern Sahara: Status, economic significance and cultural reflection in antiquity. Proceedings of an interdisciplinary ACACIA workshop held at the University of Cologne December 14-15, 2007 (pp. 293-307). Colloquium Africanum 4. HeinrichBarth-Institut. Cologne.

Huyge, D. y Claes, W. (2009). “'Ice Age’ Art along the Nile”. The Bulletin of The Egypt Exploration Society, $33,25-28$.

Kingdon, J. y Hoffman, M. (eds.) (2013). Mammals of Africa. Volume VI: Pigs, Hippopotamuses, Chevrotain, Giraffes, Deer and Bovids. Londres: Bloomsbury.

Köhler, Ch. (2002). "History or Ideology?: New Reflections on the Narmer Palette and the Nature of Foreign Relations in Predynastic Egypt”. En E. Levy y E. Van den Brink (eds.). Egypt and the Levant (pp. 499-513). Londres y Nueva York.

Lankester, F. D. (2012). Predynastic \& Pharaonic era Rock-Art in Egypt's Central Eastern Desert: Distribution, Dating \& Interpretation. Durham: Durham E-Theses. Disponible en línea en http://etheses.dur.ac.uk/5909/ (consultado el 07/11/2017).

Linseele, V., van Neer, W. y Hendrickx, S. (2007). "Evidence for early cat taming in Egypt". Journal of Archaeological Science, 34, 2081-2090.

Linseele, V., van Neer, W. y Hendrickx, S. (2008). "Early cat taming in Egypt: A correction”. Journal of Archaeological Science, 35, 2672-2673.

Linseele, V., van Neer, W. y Friedman, R. (2009). "Special animals from a special place? The Fauna of HK29A at Predynastic Hierakonpolis”. Journal of the American Research Center in Egypt, 45, 105-136.

Lythgoe, A. M. y Dunham, D. (1965). The Predynastic cemetery N7000. Naqa-ed-Der. Part IV. Berkeley: University of California Publications in Egyptian Archaeology.

Manlius, N. (2000). “Biogéographie et écologie historique de l'hippopotame en Egypte”. Belgian Journal of Zoology, 130, 59-66.

Manlius, N. (2006). “Des millénaires de grands mammifères terrestres et sauvages en Égypte”. Ankh, 14/15, 2005/2006, 101-113.

Marks, A. E. (1968). "The Khormusan: an Upper Pleistocene industry in Sudanese Nubia”. En Wendorf, F. (ed.). The Prehistory of Nubia, vol. I (pp. 315-391). Dallas: Fort Burgwin Research Center and Southern Methodist University Press.

Maydana, S. (2015). “Of Hippopotami and Hawks. Some remarks on anEgyptianan Egyptian primeval rivalry”. Cahiers Caribéens d’Egyptologie, 19-20. Université des Antilles, 49-60. 
Maydana, S. (2017). "Implicancias simbólicas y sociopolíticas de las representaciones de la cacería del hipopótamo en Egipto predinástico”. Bibliotheca Augustiniana, Vol. VIII, 40-59.

Mond, R. y Myers, O. H. (1937). Cemeteries of Armant I. Egypt Exploration Society. Londres.

de Morgan, J. (1896). Recherches sur les Origines de l'Egypte. 1: L’Age de la Pierre et des Métaux. 2: Ethnographie Préhistorique et Tombeau Royal de Nagada. Paris

Morrow, M. y Morrow, M. (eds.) (2002). Desert RATS. Rock Art Topographical Survey in Egypt's Eastern Desert. Bloomsbury Summer School. Londres: University College Press.

Müller, V. (2008). “Nilpferdjagd und geköpfte Feinde - zu zwei Ikonen des Feindvernichtungsrituals”. En EM. Engel, V. Müller y U. Hartung. Zeichen aus dem Sand. Streiflichter aus Ägyptens Geschichte zu Ehren von Günther Dreyer (pp. 477-493). Menes 5. Wiesbaden: Otto-Harrasowitz.

Myers, O. H (1933). “Two Prehistoric Objects”. Journal of Egyptian Archaeology, 19, 55.

Van Neer, W. y Linseele, V. (2009). “Animal Hospital: Healed Animal Bones from HK6”. Nekhen News 21, Fall 2009, 11-12.

Van Neer, W., Udrescu, M., Linseele, V., de Cupere, B. y Friedman, R. (2015). “Traumatism in the Wild Animals kept and offered at Predynastic Hierakonpolis, Upper Egypt”. International Journal of Osteoarchaeology, 1-20.

Petrie, W. M. F. (1901). Diospolis Parva. The Cemeteries of Abadiyeh and Hu 1898-9. Special extra publication of The Egypt Exploration Fund. Londres.

Pieri, A. y Friedman, R. (2009). “Two new tombs at HK6: The hippo hunters of Hierakonpolis”. Nekhen News 21, Fall 2009, 13-14.

Plutarco (1936). Moralia. with an English Translation by Frank Cole Babbitt. Cambridge, MA: Harvard University Press - William Heinemann Ltd.

Roche, A. (2014). “Et le roi tua l'hippopotame. Enquête sur les origins d’un rite Égyptien”. Archimede, No. 1, 71-87.

Rouch, J. (1950). Bataille sur le Grand Fleuve. Film documental.

Säve-Söderbergh (1953). On Egyptian representations of hippopotamus hunting as a religious motive. Lund: C. W. K. Gleerup.

Te Velde, H. (1967). Seth, God of Confusion. Leiden: E.J. Brill.

Teeter, E. (ed.) (2011). Before the Pyramids. The Origins of Egyptian Civilization. Oriental Institute Museum Publications 33: The Oriental Institute of the University of Chicago.

Wengrow, D. (2006). The Archaeology of Early Egypt. Social Transformations in North-East Africa, c. 10.000 to 2.650 BC. Cambridge: Cambridge University Press.

Winkler, H. A. (1938). Rock-Drawings of Southern Upper Egypt I. Londres: Egypt Exploration Society.

Zajac, A. (2008). "Some remarks on the representation of so called boat procession from Wadi el-Barramiya (Eastern Desert of Egypt)”. Studies in Ancient Art and Civilization, 12, 13-20.

“Top Ten Discoveries of 2009”. Archaeology, Vol. 63, Nr. 1, January/February 2010. Disponible en línea en http://archive.archaeology.org/1001/topten/index.html (Consultado el 30/09/2016). 